\title{
Improvements to acoustic techniques for the detection of cavitation inception
}

\author{
par G. Cangioli, G. Manfrida \\ Dipartimento di Energetica "Sergio Stecco", \\ Università degli Studi di Firenze
}

\section{I 口 INTRODUCTION}

Cavitation is probably the most severe design and operational problem encountered in the field of hydraulic machinery. The development of larger and faster (high-specific-speed) units has reduced the margin with respect to cavitation-free operation. Designers are commonly faced with the problem of correctly establishing this limit; however, hydraulic machines (both pumps and turbines) typically operate to a certain extent in cavitating conditions without exhibiting any significant performance deterioration. This has severe drawbacks from the point of view of expected life and plant reliability.

With reference to pumps, the designers still rely on the very basic work of Gongwer [1], with possible improvements described by Salisbury [2] and Pearsall $[3,4,5]$, which are all referred to methods for estimating extended cavitation conditions (EC, traditionally assumed at $3 \%$ head or power loss at fixed capacity). Only in relatively recent times cavitation inception and extended cavitation curves for pumps have been identified and studied $[6,7,8,9]$. Today, the differentiation between cavitation inception (CI, which is typically detected by visual methods) and extended cavitation has been introduced in the most recent codes and standards for pump testing [10]. On the other hand, it has been demonstrated that a correlation between $\mathrm{CI}$ and EC limits is not generally possible, as there are too many design variables involved [11]. Pump manufacturers - after having observed that some of their machines experienced cavitation

\section{LIST OF SYMBOLS}

\begin{tabular}{|c|c|c|}
\hline $\mathrm{CNL}$ & \multicolumn{2}{|c|}{ Cavitation Noise Level $\quad \sqrt{\mathrm{NL}^{2}-\mathrm{NL}_{0}^{2}}$} \\
\hline Df & \multicolumn{2}{|c|}{ Frequency bandwidth } \\
\hline DNL & \multicolumn{2}{|c|}{ Time-derived Noise Level } \\
\hline f & \multicolumn{2}{|c|}{ Frequency } \\
\hline g & \multicolumn{2}{|c|}{ Gravitational acceleration } \\
\hline NL & \multicolumn{2}{|c|}{ Noise level } \\
\hline$N L^{*}=\frac{\mathrm{NL}}{1 / 2 \rho \mathrm{u}^{2}}$ & \multicolumn{2}{|c|}{ Normalized Noise Level } \\
\hline \multicolumn{3}{|c|}{$\mathrm{NPSH}=\left(\mathrm{P}_{\mathrm{u}}-\mathrm{P}_{\mathrm{v}}\right) /(\rho \mathrm{g})$} \\
\hline & \multicolumn{2}{|c|}{ Net Positive Suction } \\
\hline$P$ & \multicolumn{2}{|c|}{ Pressure } \\
\hline Q & \multicolumn{2}{|c|}{ Flow rate } \\
\hline $\mathbf{R}$ & \multicolumn{2}{|c|}{ Normalized Autocorrelation function } \\
\hline$T$ & \multicolumn{2}{|c|}{ Integral time scale } \\
\hline \multicolumn{3}{|c|}{ Peripheral speed at impeller inlet tip } \\
\hline w & \multicolumn{2}{|c|}{ Relative velocity } \\
\hline$\theta$ & \multicolumn{2}{|c|}{ Time microscale } \\
\hline$\rho$ & & Density of water \\
\hline \multicolumn{2}{|c|}{$\begin{array}{l}\rho \\
\sigma_{b}=\left(P_{1}-P_{v}\right) /\left(1 / 2 \rho w_{1}^{2}\right)\end{array}$} & Blade cavitation number \\
\hline \multicolumn{2}{|l|}{$\tau$} & Time lag \\
\hline
\end{tabular}

\section{AMÉLIORATION DES TECHNIQUES ACCOUSTIQUES POUR LA DÉTECTION DU DÉBUT DE CAVITATION}

Les machines hydrauliques modernes se trouvent fortement limitées par la cavitation, qui peut causer des dommages d'érosion, même dans des conditions pour lesquelles on est incapable d'apprécier une variation de performance. La possibilité d'identifier les conditions de début de cavitation est par conséquent de première importance, et peut trouver des applications industrielles assez généralisées si on développe des méthodes simples, fiables et économiques. Les méthodes acoustiques se prêtent fort bien à cette intention : la méthode a été mise au point en laboratoire; elle est peu intrusive, et est capable de détecter la cavitation même dans des zones où l'accès optique est impossible. Le limites principales sont traditionnellement dans la nécessité d'utiliser une eau parfaitement dégasée, avec un niveau de bruit très réduit dans le circuit. Dans cet article, on présente des méthodes d'analyse du signal qui ont permis de repousser ces limites et d'étendre considérablement le champ possible d'utilisation. 
damage well below the EC limit - are thus faced with the problem of providing answers for customers who ask for tests ensuring cavitation-free operation under the expected operating conditions, and are very interested in techniques directly applicable on the field. Similar problems are encountered in the field of hydraulic turbines.

Direct observation of unsteady bubble growth patterns with the use of stroboscopic illumination allows to detect cavitation at the early (inception) stage [12]. However, this technique is typically applied in the laboratory and requires significant modifications to the turbomachine for optical access ; furthermore, it can be applied only to transparent liquids. The visual approach is very powerful, yet it requires a large extent of human expertise, and is thus liable to significant errors if applied on a quantitative basis. To this end, video recording and digital image processing [13] can be used as a tool for introducing standard processing techniques and allowing thus comparisons between different cavitation inception modes (e.g., with variable flowrate ; or by different impeller geometries). Actually, the measurements which are presented in this paper rely on this advanced image-recording and processing system in order to identify where possible - cavitation condition at inception on each singular blade of the impeller.

\subsection{Acoustic detection of cavitation inception}

Acoustic techniques have been applied to cavitation detection since the first work of Varga et al. [14]. Acoustic cavitation noise contains a wide range of high frequencies : from the audible range to an expected $200 \mathrm{kHz}$ or more. These techniques started with the use of microphones or accelerometers, and audio-frequency RMS noise meters or spectrum analyzers ; nowadays, they are typically applied with piezoelectric pressure or noise emission sensors [12, 15]. However, acoustic techniques are typically applied in laboratory circuits, where a low noise level can be ensured by the use of specialized devices (valves, filters, flow straighteners, etc.), and water can be deaerated at a very large extent. In fact, cavitation noise can be irradiated from other sources in the circuit (e.g., valves, elbows, etc.); and a high gas content inside the liquid produces typically "flat" noise curves [12] which do not show any peak at the inception stage, but only a decrement under conditions of extended cavitation, on account of the damping of the pressure fluctuations by gas cavities. These are the typical operating conditions on the field, and this is the main reason why acoustic techniques have not yet become very popular.

The present work tries to extend these operational limits, to include typical plant conditions where the noise level can be very high and the gas content in the liquid is large and possibly unpredicted. In order to enhance the sensitivity of acoustic analysis, bandwidth analysis techniques, methods for signal time scale reconstruction, and derivation of the signal in the frequency spectrum or time domain are applied. Many of these techniques - with special reference to this last - have provided interesting results on the test bench at the University of Florence.

The approach described in the present work tries to take full advantage of the present availability of high-performance portable spectrum analyzers. The instrumentation used was basically composed of the following :

1) B\&K Hydrophone 8103. This is actually a small (less than $10 \mathrm{~mm}$ in diameter) and very sensitive $(0,098 \mathrm{pC} / \mathrm{Pa})$ piezoelectric pressure transducer for underwater applications. The sensor frequency response extends up to $180 \mathrm{kHz}$.
2) Charge amplifier ; here a B\&K 2635 unit was used, taking advantage of the in-built low-pass and high-pass filters (settings of respectively 2 and $100 \mathrm{kHz}$ were used).

3) An ONO-SOKKI CF-5220 spectrum analyzer, having a frequency response of $100 \mathrm{kHz}$, a dynamic range of about $90 \mathrm{~dB}$.

Part of this measurement chain (charge amplifier and spectrum analyzer) is typically used in on-site vibrational tests on the field, with the simple addition of one or more accelerometers. This makes the present techniques particularly suited for field applications.

Samples of 2048 data were taken and spectra averaged over 64 samples, with triggering synchronized with shaft revolution. A sampling frequency of $256 \mathrm{kHz}$ was used, thus providing a useful sample duration of $8 \mathrm{~ms}$.

The basic innovation with respect to traditional techniques is the processing of the power spectrum records. Rather than simply calculating the RMS of the noise signal, data processing techniques developed for turbulence analysis were used. These techniques include the calculation of the time turbulence scales from the autocorrelation function, and the derivation of the power spectrum.

The data processing was done in real time by remote control of the spectrum analyzer through the GPIB interface, by means of a dedicated software. This allowed the calculation of all the parameters used for the description of cavitation inception by acoustic measurements immediately after each test point. The test operator was then free to select other pump operating points, in order to have a detailed coverage of the cavitation curve.

\subsection{Experimental facility}

All tests described in the present work have been run on the pump test facility of the D.E.F. (Department of Energy Engineering, University of Firenze). This facility [16] is an open-circuit test site with an about $300 \mathrm{~m}^{3}$ water inventory, and a variable-speed DC drive of $250 \mathrm{~kW}$. On this circuit tests have been run on an instrumented centrifugal pump prototype by Nuovo Pignone S.p.A.. This pump has a six-bladed impeller of about $400 \mathrm{~mm}$ diameter and a specific speed of 29 (S.I. units); the pump features a transparent inlet section of about 4 inlet diameters, which allows optical access for lighting by means of a high-quality strobe lamp, as well as for visual inspection. A miniature camera was used for this last purpose : the image is stored on 8-mm or VHS format, and can be then off-line treated by digital image processing in order to enhance cavity properties, picture definition, etc.

As the circuit is of the open type, cavitation is achieved by means of inlet throttling ; the capacity is kept constant by simultaneous opening of the valve located at pump discharge. The pump was tested at capacities ranging from 0,43 to $1,15 \mathrm{Q}_{\text {BEP }}$ at a rotational speed of $1000 \mathrm{rpm}$, corresponding to $0,66 \mathrm{~N}_{\mathrm{opt}}$; at this speed, the blade passing frequency is $100 \mathrm{~Hz}$, and the associated period $10 \mathrm{~ms}$. This means that the $8 \mathrm{~ms}$ sample, triggered with rotor revolution, was able to cover nearly one blade-toblade passage. The high value of the capacity, compared with the size of the inventory, and the presence of a large free surface, allows to consider the water saturated with air (free-oxygen measurements confirmed this situation).

\subsection{Definition of parameters}

Cavitation in hydraulic machines is typically studied by 


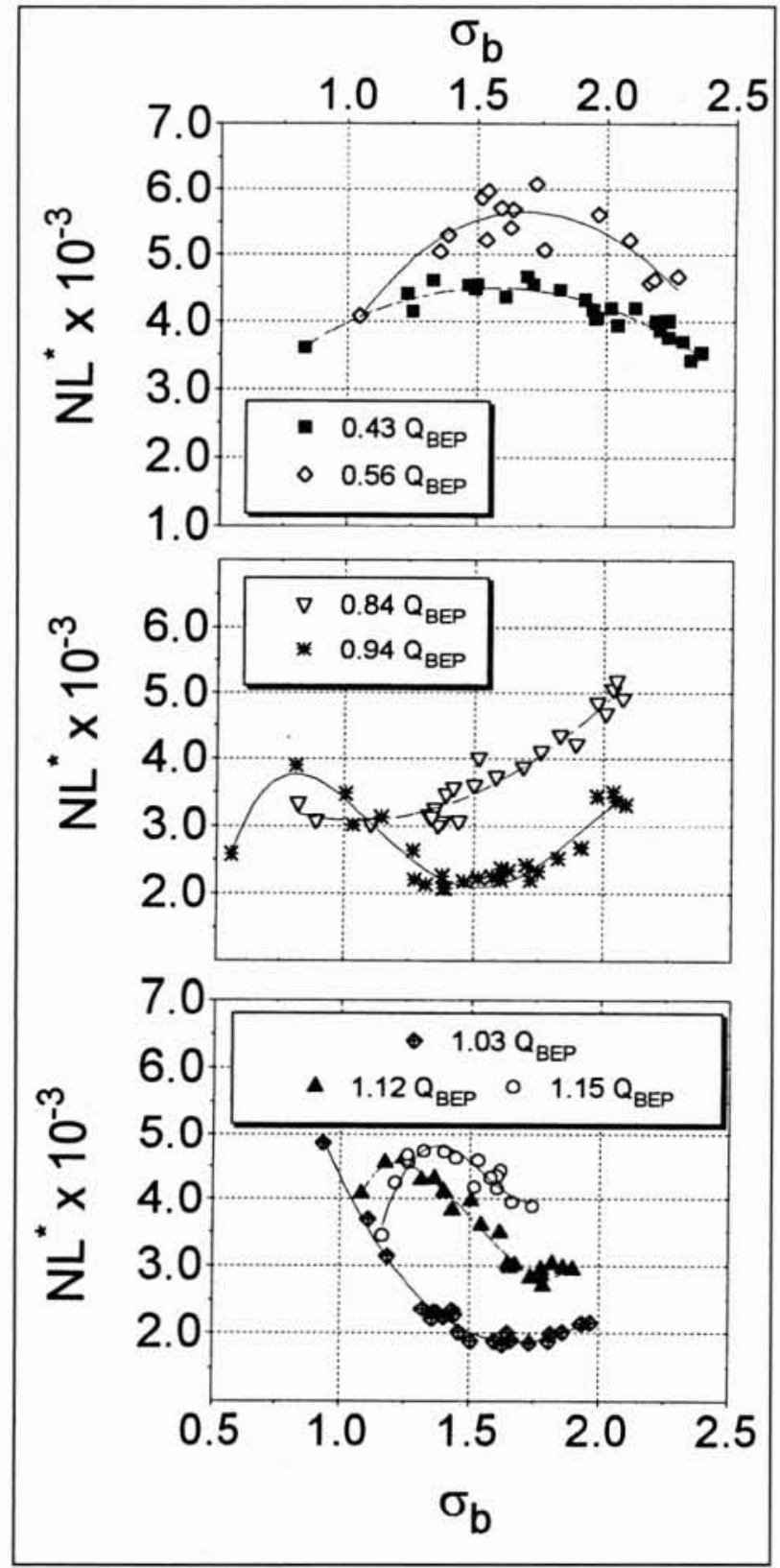

1. NL* vs. $\sigma_{b}$ for the tested pump at different capacities.

means of suitable non-dimensional parameters. Here, the blade cavitation number $\sigma_{\mathrm{b}}=\left(\mathrm{P}_{1}-\mathrm{P}_{\mathrm{v}}\right) /\left(1 / 2 \rho \mathrm{w}_{1}{ }^{2}\right)$ is used as a widely recognized indicator of cavitation development ; the traditional number used in acoustic measurements of cavitation inception is the non-dimensional noise level $\mathrm{NL}^{*}=\mathrm{NL} /\left(1 / 2 \rho \mathrm{u}^{2}\right)$. Gulich [12] also introduces a corrected noise level CNL $=\sqrt{ } \bar{N} L^{2}-N_{o}{ }^{2}$ which can be more useful than NL as it subtracts the background noise level NL $\mathrm{N}_{\mathrm{o}}$. However, the use of RMS noise levels without further analysis of the signal (apart from an analogue high-pass filter setting which can often be used for the masking of background noise and the improvement of sensitivity in the high-frequency domain, characteristic of cavitation inception noise) renders impossible to apply these techniques to systems having large background noise levels and/or limited amounts of cavitation noise (because of noise attenuation in the gas phase, on account of the large gas content of the liquid).

Special techniques of spectral signal analysis have been introduced in previous works dealing with the herein tested pump
$[17,18,19]$. These techniques have often provided satisfactory results and are hereafter briefly summarized.

The calculation of time scales, which is a common practice in turbulence studies [20,21,22, 23], can be of great help to identify cavitation conditions. The time integral scale $\mathrm{T}$ is calculated from the normalized autocorrelation function $\mathrm{R}$ :

$$
\mathrm{T}=\int_{0}^{\infty} \mathbf{R}(\tau) \mathbf{d} \tau=\sum_{k=1}^{\infty} \mathbf{R}_{k} \Delta \tau
$$

The fastest way for the calculation of the autocorrelation function is by the inverse-FFT transform of the power spectrum $[24,25]$. The autocorrelation function is also useful for the estimate of the time microscale $\theta$ :

$$
\left.\frac{d^{2} R}{d \tau^{2}}\right|_{r=0}=-\frac{2}{\theta^{2}}
$$

When directly applied to the noise signal (hydrophone output in our case), significant trends can be identified in most tests for both $\mathrm{T}$ and $\theta$ : typically, with diminishing $\sigma_{\mathrm{b}}$ (i.e., increasing cavitation), a minimum in $\mathrm{T}$ is found (C.I.), followed by a strong increase when large cavities start to be formed (E.C.) ; on the other hand, bubble nucleation and C.I. can be often identified with minimum conditions for $\theta$. This looks promising but - even if this trend was confirmed by most tests - there were admittedly situations when data interpretation was not completely sure (e.g., the maximum or minimum conditions could not be clearly identified, or where too broad to allow a precise determination of C.I.). A significant improvement was obtained in the last tests by extending the application of time scale calculations to the derivative signal, which has allowed further selectivity in the recognition of different cavitation inception modes. Early demonstrations of these techniques [17] used simpler instrumentation and implemented signal analysis by software (MATLAB) treatment of spectral data ; presently all calculations are performed in fast and reliable mode by the spectrum analyzer, which is controlled and programmed via GPIB.

The most significant improvement was found to be obtainable by derivation of the noise signal : the operation can be thus identified as the introduction of a large progressive amplification at high frequencies, which is very beneficial in the case of cavitation noise, as a flat, high-frequency power spectrum is one of the qualitative indicators of cavitation occurrence. After derivation, a Derivative Noise Level DNL can be calculated (it is the integral of the power spectrum of derivative signal, by Parseval's theorem). This was found to be a very satisfactory indicator of cavitation inception conditions, as its rise was always identified with the visual observation (where possible) of C.I.

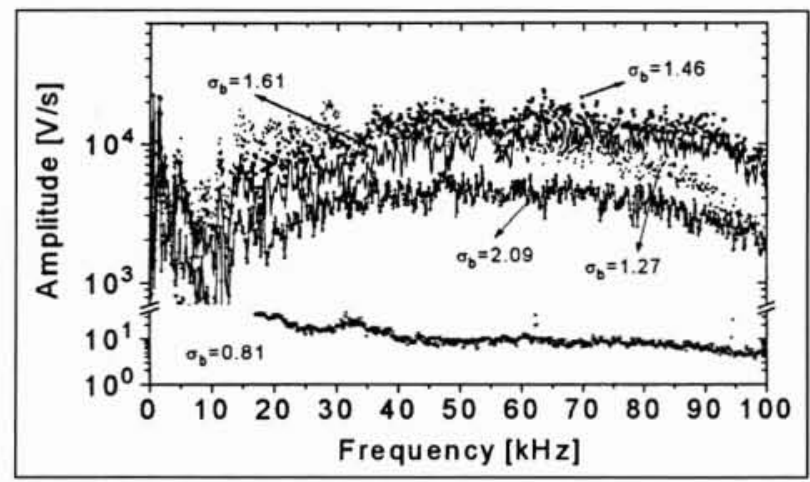

2. Noise power spectrum for different $\sigma_{b} s ; Q=0.94$. 


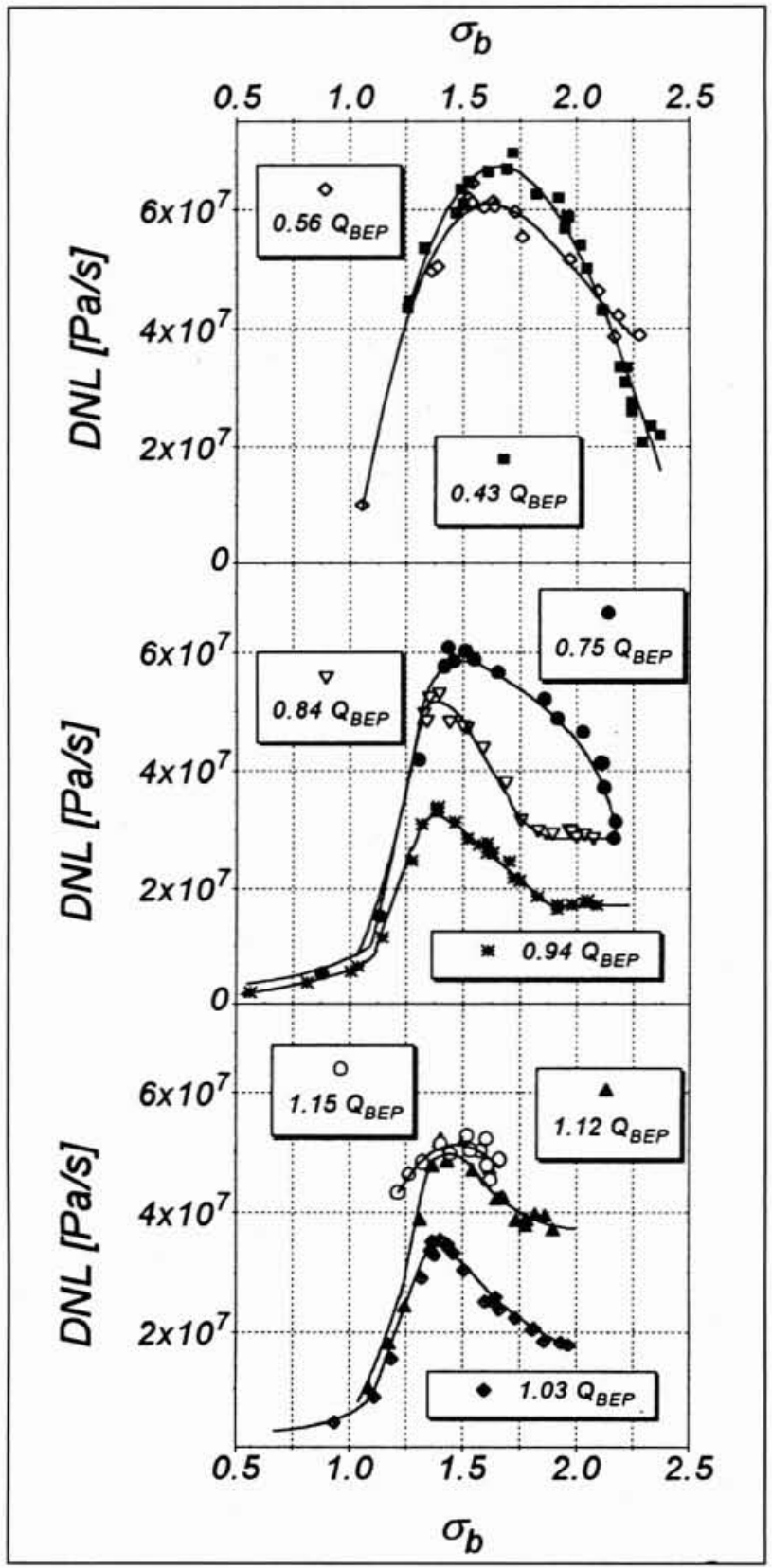

3. Derivative noise level curves versus $\sigma_{b}$ for different flowrates.

In the last test a neural network reconstruction of cavitation inception, based on the use of the new parameters introduced, was attempted in order to possibly investigate the possibility of building expert systems capable of tracking cavitation inception under current operation of hydraulic machinery.

\section{RESULTS OF THE MEASUREMENTS}

The results hereafter presented are aimed to a comprehensive presentation of the cavitation behaviour of the tested pump, and should provide a clear idea of the signal enhancement which can be gained by application of the advanced signal analysis techniques which have been presented.

The tested pump showed rather flat NL* curves (Figure 1), which are typical of circuits with large background noise and uncontrolled gas content in the liquid. Moreover, for some flo- wrates (e.g., $Q / Q_{\text {सЕP }}=0,84$ and 1.03) it was not even possible to identify a maximum in NL* vs. $\sigma_{\mathrm{b}}$ curve. These results were very discouraging.

A qualitative analysis of the noise power spectra allows a qualitative identification of C.I. inception conditions (Figure 2) : the condition $\sigma_{b}=1,46$ corresponds visually to cavitation inception ; it also provides the maximum value of noise level in the high-frequency range.

In terms of DNL, the trend vs. $\sigma_{\mathrm{b}}$ for different values of capacity is represented in Figure 3. The conditions of maximum $\sigma_{\mathrm{b}}$ were always identified (where possible) with visual detection of cavitation inception. The decrease in DNL with increasing cavitation (lower $\sigma_{\mathrm{b}} \mathrm{s}$ ) is particularly abrupt.

The application of time scale calculations to the noise signal and to its derivative is shown in Figures 4 ( $a$ and $b$ ) and 5 ( $a$ and b), respectively for macroscales $\mathrm{T}$ and microscales $\theta$. The identification of cavitation inception is difficult working on the noise signal (curves a) ; selectivity is clearly gained working on its time derivative (curves b) and calculation of time scales seems to be capable to identify the very conditions of bubble nucleation, when cavitation is still not even discernible by normal visual observation methods either by the maximum in DNL or by the qualitative analysis of the power spectrum.

A frequency-band decomposition of the noise [19] has clearly confirmed that cavitation inception corresponds to a maximum noise in the high-frequency range $(40<\mathrm{f}<100 \mathrm{kHz})$. However, in the present application, this signal (of the order of $+10 \mathrm{~dB}$ with reference to the base noise level in this frequency range) is hidden by the noise level in lower frequency bands, which is up to $30 \mathrm{~dB}$ larger. This feature is resumed by the data represented in Figure 6 ; here, the value of $\sigma_{b}$ corresponding to maximum noise emission for different frequency bands is shown vs. frequency, in the range from 5 to $100 \mathrm{kHz}$, for different flowrates. All the curves - with the notable exception of very low capacities - show a sigmoidal profile, with maximization of $\sigma_{b}{ }^{\max }$ in the high frequency range, corresponding to conditions of visual cavitation inception ; noise emission clearly shifts to lower frequencies when cavitation is more extended. As an example, with reference to the curve for $\mathrm{Q}=0,94 \mathrm{Q}_{\mathrm{BEP}}$, cavitation inception can be clearly identified at $\sigma_{b}^{\max }=1,4$; maximum noise emission shifts to frequencies lower than $25 \mathrm{kHz}$ with extended cavitation $\left(\sigma_{t}{ }^{\operatorname{ma}}<1,25\right)$. The analysis in terms of $\sigma_{\mathrm{b}}{ }^{\max }$ yields interesting results also for some special cases : for the minimum flowrate investigated, $Q=0,42 Q_{\mathrm{BEP}}$, operation of the pump was possible with or without extensive prerotation at inlet (depending on the operating point being reached from lower or larger flowrates).

\section{III $\square$ CONCLUSIONS}

The extensive test set over this particular pump and circuit has allowed some advances in the field of acoustic detection of cavitation inception :

a) The use of original signal processing techniques (calculation of time scales from autocorrelation of the acoustic emission ; differentiation of the signal from the power spectrum ; frequency-band decomposition of the signal) has allowed the application of acoustic techniques to a situation which was usually considered unapproachable by this method (mainly because of the large gas content inside the liquid and of the large low-frequency noise level in the circuit).

b) A basic premise to the success of the applied technique is the 
use of a sensitive transducer (a small hydrophone was used in the present case) and of a modern spectrum analyser with high dynamic range (of the order of $90 \mathrm{~dB}$ ) and advanced processing capabilities

c) The digital signal processing could be substituted for industrial applications by a relatively simple analog measurement chain coupled to a high-frequency pulse counter

d) Among the several global indicators of cavitation inception which were experimented, the DNL (Time-Derived Noise Level) seems to be the most selective.

However, also micro- and macroscales of the time-derived signal are very selective ; the same holds for $\sigma_{\mathrm{b}}{ }^{\max }$, especially if flowrate is also considered as a parameter for adjustment of the frequency range to be considered. On the whole, considering the multiplicity of indicators of cavitation inception which have been introduced, the most productive results could be obtained by the development of some kind of expert system capable of considering a complex set of variables.

Such a system could also provide basic information about particular cavitation modes (e.g., suction side or pressure side cavitation; onset of recirculation in pumps;...). A preliminary deve-

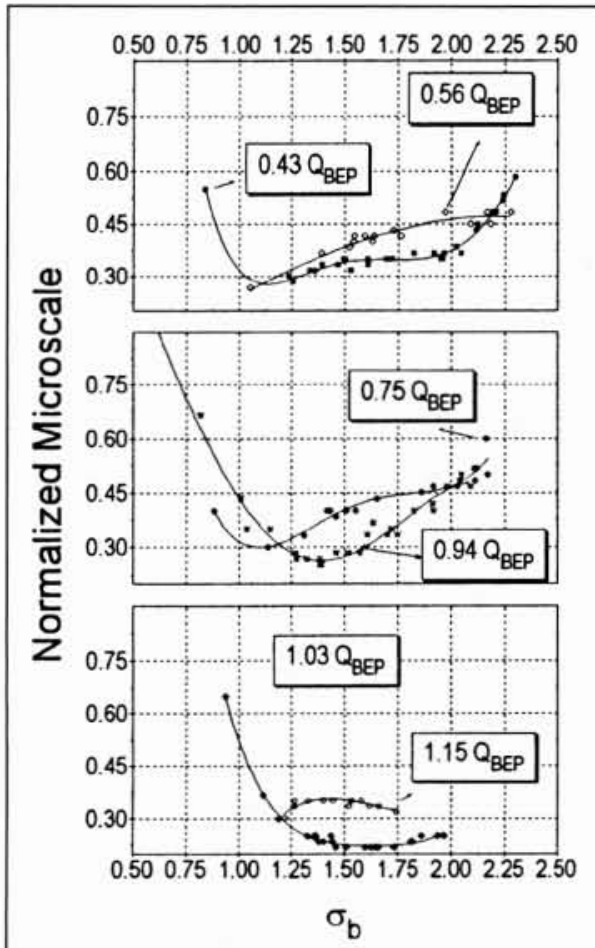

(a) Acoustic signal

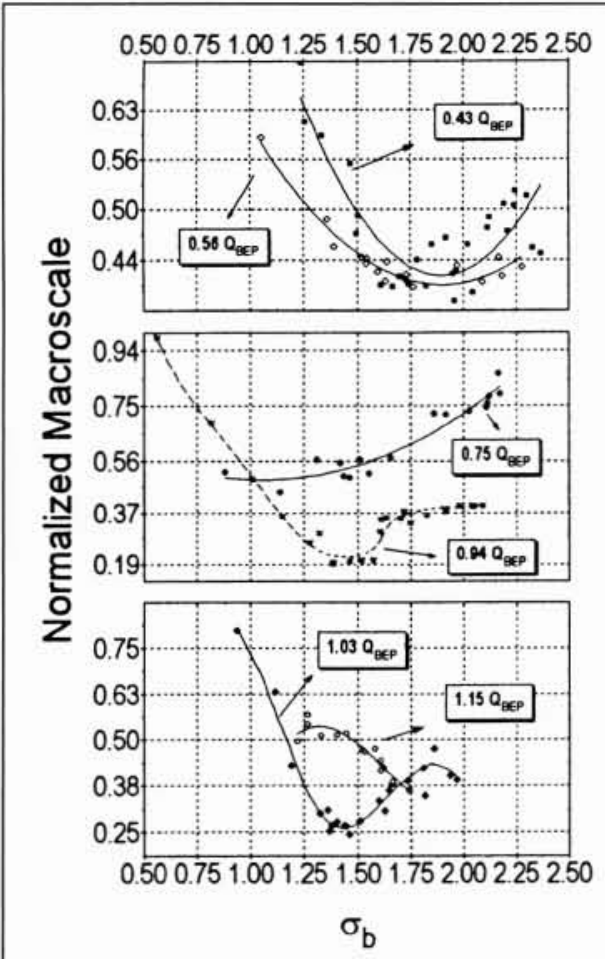

(a) Acoustic signal

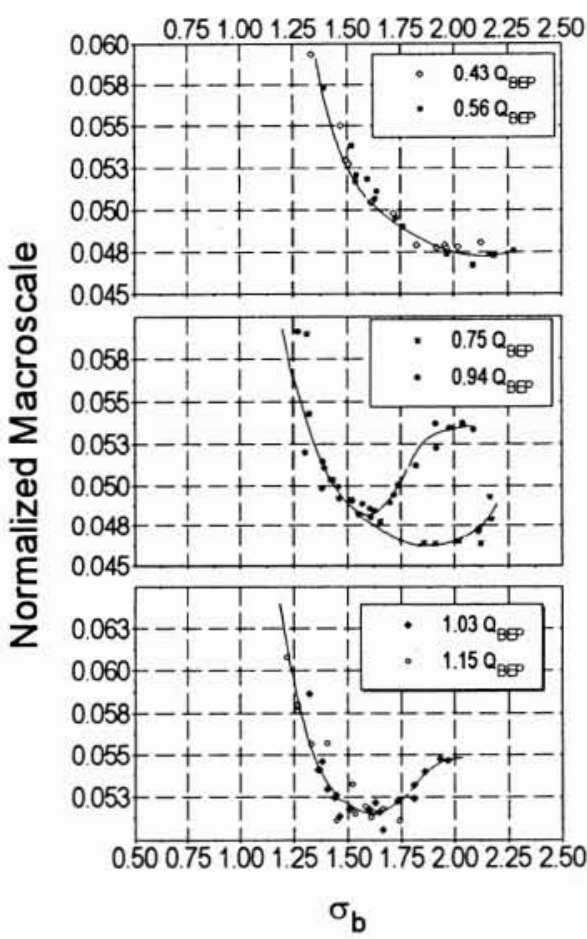

(b) Derivative of acoustic signal

\section{Integral time scales $T$ versus $\sigma_{b}$ for different flowrates.}

lopment by means of elementary neural networking techniques was done with reference to the DNL parameter alone [26]. The results (Figure 7) indicate a good capability of reproducing the experimental curves, which could be further improved by including other parameters for the recognition of cavitation inception. A three-layer back-propagating network of 21 nodes was used in the present case.

\section{ACKNOWLEDGMENTS}

This research was partially funded by the Italian Ministry of University and Research in the frame of a national project (MURST $40 \%$ ). Mr. F. Barbetti and Dr. N.Bettagli were of great help in running many tests. Nuovo Pignone S.p.A. contributed substantially with the instrumented pump and test rig buildup. $\quad \rightarrow$

5. Time microscales $q$ versus $\sigma_{b}$ for different flowrates. 


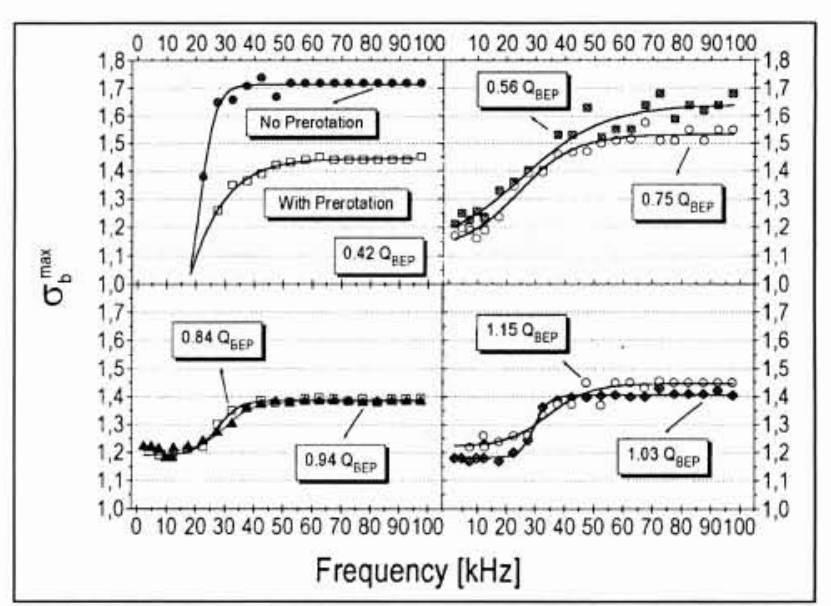

6. $\sigma_{\mathrm{b}}$ corresponding to maximum noise emission vs. frequency, for different flowrates.

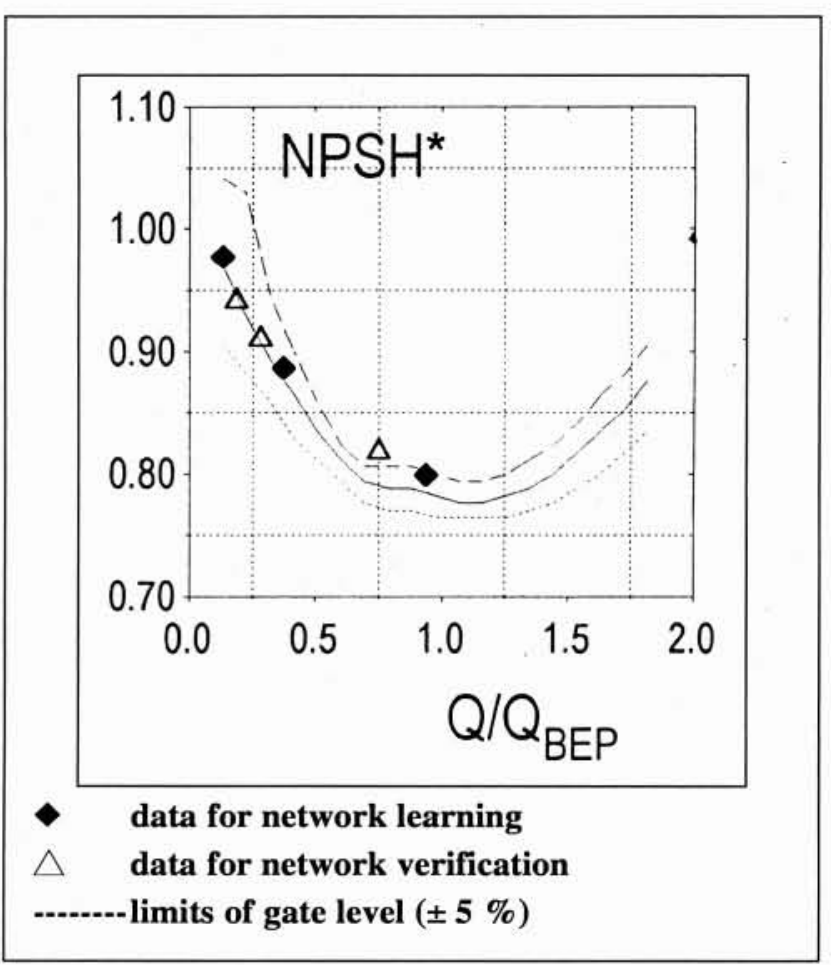

7. Reconstruction of the NPSH curve by means of neural networking applied to the DNL parameter (gate level for DNL variation $5 \%$ ).

\begin{tabular}{ll}
\hline \multicolumn{2}{l}{ Subscripts and superscripts: } \\
BEP & Best Efficiency Point \\
max & Maximum noise condition \\
opt & Project value \\
t & Total conditions \\
v & Refers to vapor \\
1 & Refers to impeller inlet \\
$*$ & Normalized
\end{tabular}

\section{RÉFÉRENCES}

[1] GoNGWER, C.A., 1941, "A theory of cavitation flow in centrifugal pump impellers", Trans. ASME, 63, 29-40.

[2] SAlusbury, A.G., 1983, "Current concepts in centrifugal pump hydraulic design", Proc. Inst. Mech. Engrs, 197,A, pp. 221-231.

[3] Pearsalt, I.S., 1973, "Design of pump impellers for optimum cavitation performance", Proc. Inst. Mech. Engrs, 187, 55/73, pp. 667-679.

[4] Pearsall, I.S., 1974, "Cavitation", IMECH-E, CME, July 1974, pp. 79-85.

[5] Pearsall, I.S., 1978, "Off-design performance of pumps", VKI LS 1978-3

[6] Canavelis, R., Grison,P., 1986, "Aspects industriels de la cavitation dans les pompes" - Revue Française de Mécanique $n^{\circ} 1986-4$

[7] GurTon, P., "Actual behaviour of pumps outside their high efficiency range" - Von Karman Institute for Fluid Dynamics, Lecture series 1978-3

[8] Ross, R., "Theoretical prediction of net positive suction head required (NPSHR) for cavitation free operation of centrifugal pumps", UCP.

[9] VLAMING, D.J., 1981, "A method for estimating the net positive suction head required by centrifugal pumps", ASME Paper 81-WA/FE-32.

[10] ASME, 1990,"Centrifugal Pumps", ASME PTC 8.2

[11] Sel.m, S.M.A., Khalifa, B.A., Hosien, 1989, "Cavitation inception in centrifugal pumps", ASME International Symposium on Cavitation Inception, San Francisco.

[12] GuLICH,J.F., 1989, "Guidelines for prevention of cavitation in centrifugal pumps" , EPRI GS-6398, 1989

[13] DE LuCIA, M., ANGuzzA, G., 1994, "Visualization and image processing to study cavitation process in Hydraulic Turbomachinery", Modelling, Testing \& Monitoring for Hydro-Power Plants, Budapest

[14] VArGa, J.J., Sebestyen, G., FAy, A., 1969, "Detection of cavitation by acoustic and vibration-measurement methods", La Houille Blanche, 2, pp. 137. 149.

[15] Leduce, D., Wegner, M., 1985, "Méthodes d'approche du bruit engendré par la cavitation", La Houille Blanche, 8, pp. 697-708.

[16] Arnone, A., Bosio, A., Carnevale, E., De Lucia, M., Facchini, B., MANFridA, G., 1991, "Il banco prova pompe del Dipartimento di Energetica dell'Università degli Studi di Firenze", 46th ATI Congress, Gaeta.

[17] Cangioli, G., Manfrida, G., Ranieri, P., 1995, "New Techniques for the Acoustic Detection of Cavitation Inception in Hydraulic Machinery", 14th BPMA Technical Conference, Birmingham, U.K.

[18] Cangioli, G., Manfrida, G., 1995, "Signal Proccesing Techniques for the Diagnosis of Cavitation Inception" JSME-ASME Symposium on Cavitation and Gas-Liquid Flow in Fluid Machinery and Devices, Hilton Head.

[19] Cangiou, G., 1995, "Characterization of cavitation in a Centrifugal Pump through Maximum Noise Analysis", International Symposium on TwoPhase Flow Modelling and Experimentation, Roma .

[20] Bradshaw, P., 1971, "An Introduction to Turbulence and its Measurement", Pergamon Press, Oxford.

[21] Rasmussen, C.G., 1966, "Measurement of Turbulence Characteristics", DISA Information n.6.

[22] TENNEKES, H., LUMLEY J.L., 1972, "A First Course in Turbulence", MTT Press

[23] HiNZE, J.O., 1975, "Turbulence", Mc Graw-Hill.

[24] BENDAT, PIERSOL, 1971, "Random Data: Analysis and measurements procedures", Wiley-Interscience

[25] Oppenheim A.V., Schafer R.W., 1975, "Digital Signal Processing" , Prentice-Hall Inc

[26] G.CANGioli, 1996, "Nuove metodologie per il rilevamento acustico della cavitazione in pompe idrauliche", PhD Thesis, Università degli Studi di Firenze. 